\title{
BENEFICIOS DE LAS INTERVENCIONES DE TERAPIA OCUPACIONAL EN LA REDUCCIÓN DE SÍNTOMAS DEPRESIVOS EN PERSONAS MAYORES DE 60 AÑOS
}

\author{
BENEFITS OF OCCUPATIONAL THERAPY INTERVENTIONS IN REDUCTION OF \\ DEPRESSIVE SYMPTOMS IN PEOPLE OVER 60 YEARS OLD
}

\section{Eva Álamo Gutiérrez ${ }^{1}$}

\begin{abstract}
RESUMEN
Objetivo: Esta revisión sistemática tiene como objetivo describir los beneficios que tienen las intervenciones de Terapia Ocupacional en la reducción de los síntomas depresivos en personas mayores de 6 o años. Metodología: se realizó una búsqueda en diferentes bases de datos (PubMed, ScienceDirect, CINAHL, OTseeker, OTDBASE, Cochrane, PsycINFO, Trip Database y Jábega 2.o.), seleccionando artículos publicados entre el año 2000 y 2016, tanto con diseño cualitativo como cuantitativo. Resultados: Las intervenciones realizadas (revisión de historia de vida, uso de actividades de ocio y tiempo libre, redefinición de estilos de vida saludables, el buen manejo de la enfermedad e intervenciones cognitivas y de psicoeducación) logran una mejoría de la sintomatología depresiva. Conclusión: Las intervenciones realizadas desde Terapia Ocupacional son beneficiosas para reducir los síntomas depresivos en personas mayores de 6o años.
\end{abstract}

\section{PALABRAS CLAVE}

Depresión, Anciano, Geriatría, Terapia Ocupacional

\begin{abstract}
Introduction: This systematic review has the aim of describing the benefits of the Occupational Therapy's interventions in the reduction of depressive symptoms in people over 60 years. Methodology: A search was conducted in different research databases (PubMed, ScienceDirect, CINAHL, OTseeker, OTDBASE, Cochrane, PSyCINFO, Trip Database y Jábega 2.o.), selecting articles published between 2000 and 2016, both with qualitative and quantitative design. Results: The interventions carried out (review of life history, use of leisure and free time activities, redefinition of healthy lifestyles, good management of the disease and cognitive and psychoeducation interventions) achieve an improvement in depressive symptomatology. Conclusion: The interventions made from Occupational Therapy are beneficial to reduce depressive symptoms in people over 60 years.
\end{abstract}

\section{KEYWORDS}

Depression, Aged, Geriatric, Occupational Therapy

Recibido: 29 Agosto 2018

Aceptado: 30 Septiembre 2019

1 Terapeuta ocupacional. Universidad de Málaga. Terapeuta Ocupacional en Residencia Municipal de Mayores Fernando Santos Calle Lope de Vega, 2214412 Pedroche (Córdoba). ResearchedID: T-5847-2018 ORCID ID: https://orcid.org/0000-0002-2913-6783. España. 610832705 eva-alamo@hotmail.com 


\section{INTRODUCCIÓN}

La depresión se define como "un estado de hipertimia dolorosa, mediante el cual la persona siente una tristeza profunda que afecta a su funcionalidad, relaciones, valores y equilibrio fisiológico o como un sentimiento patológico de tristeza" (Sevilla \& Dallasta, 2006).

No siempre existe un diagnóstico de depresión en el anciano, sino que estas personas llegan a experimentar síntomas depresivos sin necesidad de que exista tal diagnóstico (Haltiwanger \& Galindo, 2013).

Los ancianos deprimidos experimentan síntomas depresivos de forma muy diferente, pudiéndolos resumir según Sevilla \& Dallasta (2006) en: síntomas emocionales (abatimiento, tristeza, llanto, irritabilidad, miedo, ansiedad, soledad, sensación de inutilidad y fracaso), síntomas cognitivos (pensamientos suicidas, ideas delirantes, pesimismo, alucinaciones, fallos de memoria y concentración), síntomas físicos (anorexia, astenia, estreñimiento, trastornos del sueño, pérdida de motivación y de la libido), síntomas relacionados con el aspecto (expresión triste, postura inmóvil, aspecto desaliñado y retraimiento social), síntomas psicomotores (movimientos lentos, marcha inquieta, falta de gesticulación, bradilalia) y comportamientos inadecuados (conductas agresivas, negativas y suicidas).

En las personas mayores aparecen trastornos mentales con más facilidad y esto se debe principalmente a la interacción de factores como el deterioro físico y funcional del cerebro, los cambios en los sentidos sensoriales, en la personalidad y los cambios psicosociales (Sevilla \& Dallasta, 2006). Así pues, la depresión, junto con la demencia, es la enfermedad más frecuentes en las personas mayores (Ayala, 2007). Se calcula que entre el $10 \%$ y el $45 \%$ de las personas mayores presentan en algún momento algún síntoma depresivo (Sevilla \& Dallasta, 2006).

Entre los factores de riesgo (sufrir alteraciones del sueño, ser mujer, tener antecedentes de depresión, sufrir algún tipo de discapacidad o estar pasando por un proceso de duelo) que tienen las personas mayores de 60 para padecer síntomas depresivos, también se encuentra la institucionalización en residencias geriátricas; debiéndose principalmente a factores como el sentimiento de abandono, cambios en los estilos de vida, dificultad de adaptación a la nueva situación, pérdida de intimidad, de autonomía y de libertad, sensación de minusvalía y de rol de enfermo, muerte del cónyuge y valoración como suceso negativo el ingresar en una residencia (Mora, 2001).

Los síntomas depresivos pueden impedir el funcionamiento independiente en las actividades de la vida diaria (AVD), obstaculizan la calidad de vida y pueden afectar a la esfera física de la persona que los padezca (Chippendale \& Bear-Lehman, 2012). También suelen limitar la participación en las actividades de la vida diaria instrumentales (AVDI) y se ven alteradas las habilidades, hábitos y rutinas de la persona (Leibold, Holm, Raina, Reynolds, \& Rogers, 2014). Por lo que todas estas dificultades, a consecuencia de la sintomatología depresiva, dejan ver que la intervención de Terapia Ocupacional (TO) en esta población podría ser beneficiosa.

Esta disciplina tiene un papel muy importante en evitar el deterioro tanto físico como psíquico que conlleva la etapa evolutiva de la vejez (Acebo, Mero, \& Bravo, 2016). Además, la TO está basada en la idea de que la salud y el bienestar se ven afectados por el patrón erróneo de ocupaciones al que da lugar la sintomatología depresiva que los mayores suelen padecer (Ciucurel \& Iconaru, 2012). De hecho, los niveles de depresión afectan directamente a la ocupación pues alteran la capacidad del individuo de dirigirse a un objetivo y dificultan su capacidad de desempeño en las actividades de la vida diaria (Ciucurel \& Iconaru, 2012).

Sin embargo, aunque los déficits que una persona con síntomas depresivos tiene son evidentes, y aun sabiendo que la TO es una disciplina que tiene un campo muy amplio de intervención en trastornos mentales y con la población mayor, no existen muchas investigaciones que demuestren los beneficios de sus intervenciones.

Igualmente, se entiende que la herramienta de trabajo de la TO, la ocupación, puede jugar un papel en la promoción de la salud y de esta forma, en la prevención de muchas enfermedades. Miralles (2003) afirma que la participación por parte de los usuarios en ocupaciones que son significativas para ellos, favorece su bienestar psicológico.

De esta forma, la ocupación, como agente para promover la salud y prevenir enfermedades, busca el objetivo de favorecer dichos estilos de vida saludables y así, prevenir las enfermedades somáticas y sus consecuencias 
y déficits, y de mantener y mejorar el sentimiento de bienestar promoviendo la actividad correcta de las estructuras y funciones corporales y el bienestar físico, mental y social (Miralles, 2003).

Este hecho demuestra como la ocupación puede servir como método de tratamiento para alcanzar unos objetivos, anteriormente establecidos según las necesidades y déficits de los usuarios evaluados, y poder restaurar o recuperar las capacidades o destrezas psicológicas, emocionales y relacionales que están impidiendo la ejecución ocupacional en el individuo (Miralles, 2003).

Por lo que a través de esta revisión sistemática se plantea conocer las intervenciones de TO y los beneficios de estas en la reducción de la sintomatología depresiva que puede padecer el mayor; así como la contribución de los estilos de vida saludables en la prevención de síntomas depresivos y la promoción del bienestar psicológico.

\section{MÉTOdo}

\section{Estrategia de búsqueda}

El diseño de estudio es una revisión sistemática sobre los beneficios de la TO en la reducción de la sintomatología depresiva que puede darse en personas mayores de 60 años. Esta hipótesis emerge a partir de la pregunta Population - Exposure - Outcomes (PEO) (BettanySaltikov, 2012): ¿son beneficiosas las intervenciones de TO para reducir los síntomas depresivos que pueden acontecer en personas mayores de 60 años?

Para realizar la revisión sistemática se efectuó una búsqueda en las siguientes bases de datos: PubMed, ScienceDirect, CINAHL, OTseeker, OTDBASE, Cochrane, PsycINFO, Trip Database y Jábega 2.o.

Dicha búsqueda se desarrolló durante los meses de Febrero, Marzo y Abril del año 2016 y para ello, se usaron estas palabras clave: depression, depressive symptoms, elderly, older, aged, aging, old age, older people, older adults, geriatric, late-life depression, depression in the elderly, occupational therapy, occupation-based intervention, occupation-based practice, lifestyle redesign programs o health promotion program. Cada palabra clave se buscó de forma individual incluyéndola entre comillas. Se utilizó el operador booleano AND como operador de intersección y el operador booleano OR como operador sumatorio para combinar palabras clave similares.

Los criterios de inclusión que se tuvieron en cuenta a la hora de seleccionar los artículos fueron: artículos publicados entre los años 2000 y 2016 (a excepción un artículo publicado en 1992 incluido debido a la relevancia de este relacionada con la revisión sistemática y a los pocos resultados encontrados en la búsqueda), de diseño cuantitativo o cualitativo, que respondan a la pregunta PEO, de diseño cuantitativo que superen según Cabello (2015) el instrumento de lectura crítica Critical Appraisal Skills Programme Español (CASPe), de diseño cualitativo que superen el marco de referencia específico para investigaciones cualitativas, que incluyan a participantes mayores de 60 años y que entre las variables estudiadas esté la medición de síntomas depresivos, donde se lleve a cabo una intervención desde TO, disponibles desde el repositorio de la Universidad de Málaga y escritos en inglés.

Como criterios de exclusión se tuvieron en cuenta artículos publicados con una fecha anterior al año 2000, que no incluyan a participantes mayores de 60 años $y$ que entre las variables estudiadas no esté la medición de síntomas depresivos, que no se haya llevado a cabo una intervención desde TO, que no estén disponibles desde el repositorio de la Universidad de Málaga o que estén escritos en otro idioma diferente al inglés.

\section{Resultados}

Como muestra la ilustración 1, en la fase de identificación se obtuvieron un total de 1134 registros tras realizar la búsqueda con las palabras clave en las diferentes bases de datos. En la siguiente fase, fase de cribado, después de eliminar aquellos registros que estaban duplicados, continúan 663 registros, de los cuales son excluidos 648 por no cumplir con los criterios de inclusión para realizar dicha revisión narrativa. Posteriormente, en la fase de elegibilidad, se obtienen 15 artículos para su lectura completa con el fin de analizar su elegibilidad. De estos artículos, los que contaban con diseño cuantitativo fueron analizados según el instrumento de lectura crítica CASPe y a partir de este se excluyó 1 artículo. De la misma forma, los artículos que contaban con diseño 
cualitativo fueron analizados con el marco de referencia específico para investigaciones cualitativas (Walsh \& Downe, 2006). Según este marco ningún artículo fue excluido. Así se llega a la última fase, la de inclusión, la cual cuenta con un total de 14 artículos para realizar la revisión sistemática.
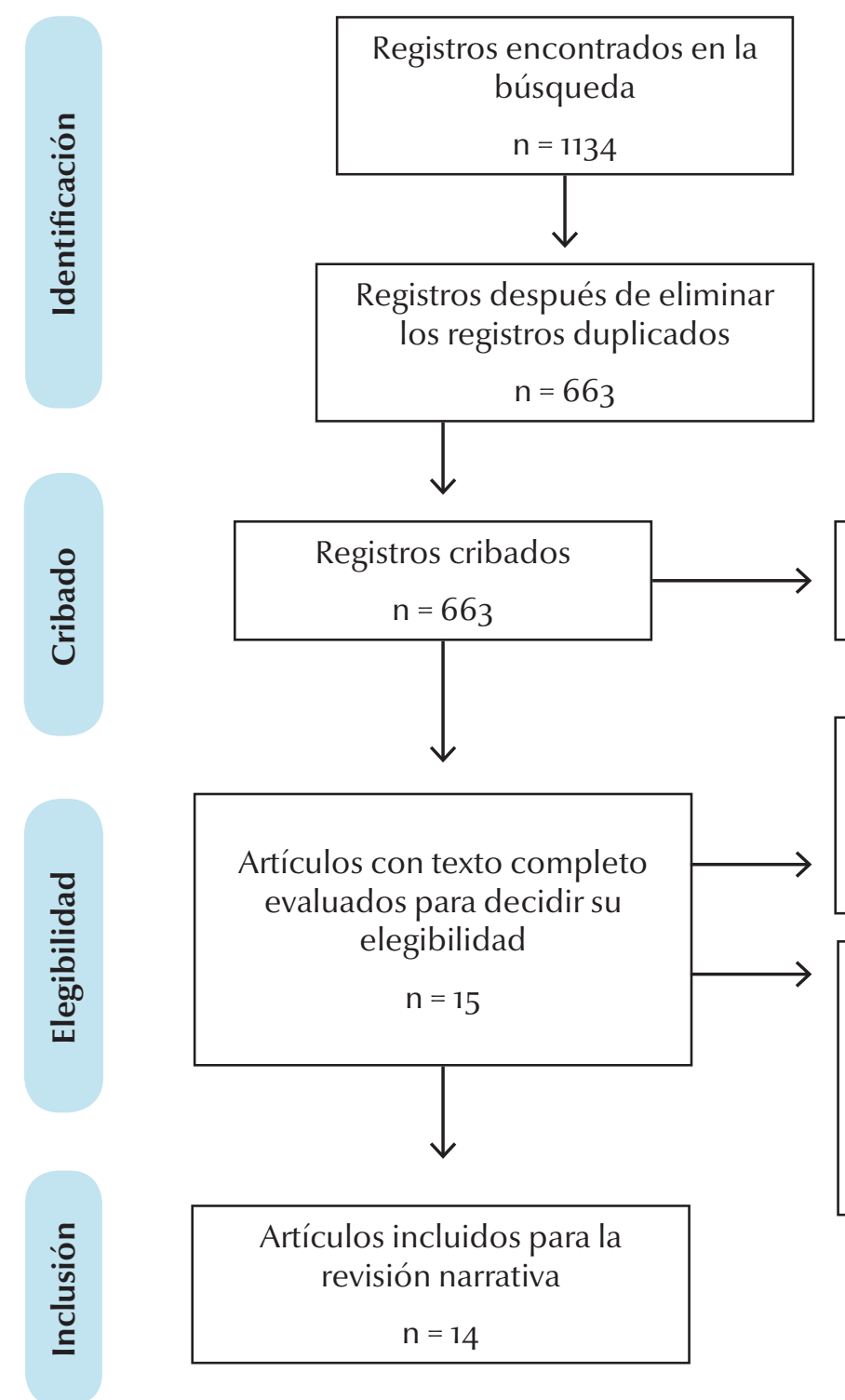

Registros excluidos

$$
\mathrm{n}=648
$$

Artículos cuantitativos con texto completo excluidos según "CASPe"

los con texto completo evaluados para decidir su elegibilidad

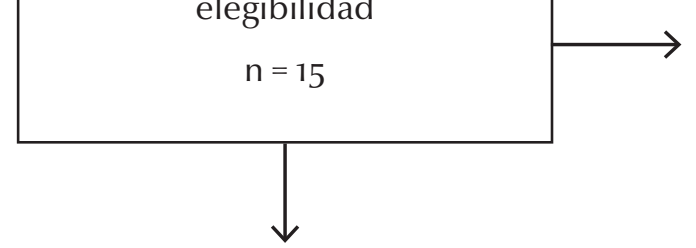

$$
\mathrm{n}=1
$$

Artículos cualitativos con texto completo excluidos según el marco para investigaciones cualitativas de Walsh \& Downe (2006)

$$
\mathrm{n}=\mathrm{O}
$$

\section{Artículos incluidos para la} revisión narrativa

$$
\mathrm{n}=14
$$

\section{FIGURA 1. DIAGRAMA DE FLUJO PRISMA DEL PROCESO DE SELECCIÓN. FUENTE: ADAPTADO DE PRISMA DIAGRAMA DE} FLUJO (MOHER, LIBERATI, TETZLAFF, ALTMAN, \& PRISMA GROUP, 2OO9). 
Para analizar los resultados y conclusiones a las que llegan los estudios se han analizado las diferentes intervenciones poniendo en común aquellas que tenían aspectos semejantes entre ellas. Este proceso se ha realizado agrupando dichas intervenciones por bloques temáticos en función de la naturaleza de cada una de ellas:

\section{Revisión de historia de vida}

Cuatro estudios usan la revisión de historia de vida como forma de tratar los síntomas depresivos que acontecen en las personas mayores. Chippendale \& Bear-Lehman (2012) desarrollan una intervención de revisión de vida haciendo uso de la escritura con el objetivo de disminuir los síntomas depresivos en las personas mayores que residen en residencias. Estos autores obtienen como resultados que la intervención es útil para reducir los síntomas depresivos y que es una buena intervención para la práctica de TO. Siguiendo con el hilo de esta intervención, otro autor, Cooper (2013) con el objetivo de conocer los beneficios de las técnicas "Usar la Escritura como Terapia" y "Escritura Creativa" en la depresión, desarrolla dos intervenciones basadas en estas técnicas. Este estudio videncia que "Usar la Escritura como Terapia" es una herramienta útil para el trabajo de síntomas depresivos en el mayor y que la revisión de historia de vida mediante la escritura es una intervención beneficiosa para reducir la depresión y muy útil para los terapeutas ocupacionales. Stevens-Ratchford (1993) vuelve a investigar el efecto de las actividades de reminiscencia en el manejo de la depresión, encontrando que esta intervención no consigue reducir de manera significativa la sintomatología depresiva. Relacionado con la forma de estructurar la intervención en períodos de vida de forma cronológica y usando el dibujo, Gunnarsson, Wagman, Håkansson, \& Hedin (2015) describen The Tree Theme Method (ElMétodo del Árbol Temático), un método de intervención psicosocial usado en TO. Aunque se trate de un protocolo y todavía no haya resultados, en línea con los dos primeros estudios, podría ser una buena intervención que ayude a la revisión de historia de vida.

\section{Ocio y tiempo libre}

Varios estudios incluidos en la revisión hacen uso de actividades relacionadas con el ocio y el tiempo libre como medio terapéutico para disminuir la sintomatología depresiva en personas mayores de 60 años. McWha,
Pachana, \& Alpass (2003) evalúan la efectividad de la actividad terapéutica grupal en mujeres mayores con depresión. Para ello, usan en la intervención actividades relacionadas con la interacción social y actividades relacionadas con el ocio y el tiempo libre. Esta intervención resultó ser útil y beneficiosa para mujeres mayores deprimidas. De la misma forma, en la revisión sistemática de Fine, (2001) varios estudios evalúan la efectividad de las actividades de ocio y tiempo libre en la reducción de los niveles de depresión. Los resultados en común de los diferentes artículos demuestran que las actividades de ocio y tiempo libre reducen los síntomas depresivos en personas mayores de 60 años. Haciendo uso de las actividades placenteras, del mismo modo que los estudios anteriores, Sood, Cisek, Zimmerman, Zaleski, \& Fillmore (2003) plantean el objetivo de comprobar la eficacia de un programa de bienestar geriátrico. Como resultados, a pesar de que las diferencias no son estadísticamente significativas, se aprecia una disminución de síntomas depresivos y una mayor percepción de salud física y mental, a la misma vez que se deja ver la intervención como una herramienta clave para la práctica de la TO en el manejo del estado anímico de los usuarios. También estudios como los liderados por Mozley et al. (2007) ponen de manifiesto la importancia de que la depresión en las residencias debería reducirse con un programa de TO, pero los resultados de este estudio declaran que el programa no tiene efectos significativos en la depresión, aunque el análisis de las entrevistas cualitativas sugiere que el programa puede ser significativo para algunos participantes.

\section{Redefinición de estilos de vida saludables}

Varios estudios buscan redefinir los estilos de vida de sus participantes para así promover estilos de vida más saludables y que eso conlleve una reducción de la sintomatología depresiva usando para ello el Lifestyle Redesign Program. Horowitz \& Chang (2003) tienen el objetivo de comprobar los efectos de este programa en la salud y en el bienestar en personas mayores de 60 años. Asimismo, Clark et al. (2012) tienen el objetivo de determinar los efectos que tiene dicho programa en el bienestar físico y mental en personas mayores de 60 años. Estos dos estudios, basados en The Well Elderly Stu$d y$, demostraron que los grupos de intervención tenían puntuaciones menores en las escalas y test que medían los niveles de depresión. Del mismo modo, Mountain, Mozley, Craig, \& Ball (2008) llevaron a cabo el Lifestyle 
Redesign Program, buscando la mejora de la salud y el bienestar de los ancianos construyendo estilos de vida más saludables. Sin embargo, este tipo de intervención no demostró tener resultados significativos en los niveles de depresión en comparación con un grupo control.

\section{Buen manejo de la enfermedad}

El estudio de Haltiwanger \& Galindo (2013) tiene el objetivo de desarrollar actividades basadas en la ocupación para disminuir los niveles de depresión en una mujer mayor diagnosticada de diabetes (estudio de caso único). Dicho estudio demostró que la intervención fue beneficiosa tanto para la reducción de los síntomas depresivos como para el mejor manejo de la diabetes.

\section{Otras intervenciones: Cognición y Psicoeducación}

Cho et al. (2015) evalúan la eficacia de un programa de entrenamiento grupal de estimulación cognitiva en la reducción de la sintomatología depresiva. Por otro lado, Tanaka, Ishikawa, Mochida, Kawano, \& Kobayashi (2015) evalúa la reducción de síntomas depresivos en personas mayores de 60 años a través de un programa de psicoeducación. Ambos estudios demuestran que sus intervenciones son útiles para reducir los síntomas depresivos. El primer estudio lo hace a pesar de no existir diferencias estadísticamente significativas entre los grupos, pero sí en el posttest del grupo de intervención, y el segundo, además, demuestra que se mejora la recuperación y la adherencia al grupo.

TABLA 1

DESCRIPCIÓN GENERAL DE LOS ESTUDIOS SELECCIONADOS

\begin{tabular}{|c|c|c|c|c|}
\hline $\begin{array}{c}\text { AUTOR / } \\
\text { LOCALIZACIÓN }\end{array}$ & OBJETIVO & $\begin{array}{c}\text { DISEÑO Y NIVEL } \\
\text { DE EVIDENCIA } \\
\text { CIENTÍFICA }\end{array}$ & $\begin{array}{l}\text { CRITERIOS DE } \\
\text { INCLUSIÓN DE LA } \\
\text { MUESTRA }\end{array}$ & $\begin{array}{l}\text { PRINCIPALES } \\
\text { CONCLUSIONES }\end{array}$ \\
\hline
\end{tabular}

\begin{tabular}{|c|c|c|}
\hline Cooper, 2013 & $\begin{array}{l}\text { Conocer los bene- } \\
\text { ficios de "Usar la } \\
\text { Escritura como } \\
\text { Terapia", la cual es } \\
\text { estructurada, breve } \\
\text { y llevada a cabo por } \\
\text { un terapeuta ocupa- } \\
\text { cional frente a "Escri- } \\
\text { tura Creativa", que } \\
\text { es desestructurada y } \\
\text { no es llevada a cabo } \\
\text { por un terapeuta } \\
\text { ocupacional }\end{array}$ & $\begin{array}{c}\text { Cualitativo } \\
\text { Ensayo } \\
\text { pretest-posttest }\end{array}$ \\
\hline
\end{tabular}

Síntomas depresivos

Edad 18-65 años

Plena disposición

Comunicación verbal

Amplia literatura

Razonamiento

No psicosis

Atención

multidisciplinar

$\mathrm{N}=24$
"Usar la Escritura como Terapia":$$
\mathrm{n}=12
$$

"Escritura Creativa":
Escritura como intervención costo-efectiva y beneficiosa para depresión, fácil de usar por la TO; "Escritura como Terapia" beneficiosa para depresión; mayor entrenamiento, clarificación e investigación de ambas técnicas

$$
\mathrm{n}=12
$$




\begin{tabular}{|c|c|}
\hline $\begin{array}{c}\text { AUTOR / } \\
\text { LOCALIZACIÓN }\end{array}$ & OBJETIVO \\
\hline $\begin{array}{l}\text { Sood et al., } 2003 \\
\text { Manhattan }\end{array}$ & $\begin{array}{l}\text { Comparar los resul- } \\
\text { tados que muestra el } \\
\text { grupo de interven- } \\
\text { ción que recibe un } \\
\text { Programa Geriátrico } \\
\text { de Bienestar frente } \\
\text { al grupo de control } \\
\text { que recibe TO estan- } \\
\text { darizada con tera- } \\
\text { peutas ocupaciona- } \\
\text { les no involucrados } \\
\text { con el programa }\end{array}$ \\
\hline
\end{tabular}

Mozley et al., 2007

Inglaterra
Comprobar la hipótesis de que la depresión en personas mayores en residencias se debería reducir con un programa de TO

\section{Cuantitativo y cualitativo}

Ensayo con grupo de control, no aleatorizado

Nivel 2 de evidencia científica
Usuarios de residencias en propiedad o anteriormente en propiedad local Ocupadas por $\geq 25$ usuarios

No especializadas

No trastorno afectivo mayor $\mathrm{N}=143$

Grupo de control

$$
\mathrm{n}=65
$$

Grupo de intervención

$$
\mathrm{n}=78
$$

\section{Cuantitativo y Mujer mexicana cualitativo \\ Caso único 62 años}

México

Diagnóstico de dia-

\begin{abstract}
mejoran el automa-
Determinar si las actividades basadas en la ocupación nejo de la diabetes y reducen los síntomas depresivos
\end{abstract}

Haltiwanger \& Galindo, 2013
PRINCIPALES CONCLUSIONES

Diferencias no estadísticamente significativas entre grupos en la percepción de la salud física o mental y en los niveles de depresión; disminución de síntomas depresivos, mayor percepción de salud física y mental en el grupo de intervención; el estado anímico mejora con la TO

No efectos estadísticamente significativos de la TO en la depresión ni en el resto de variables cuantitativas; según las entrevistas cualitativas intervención beneficiosa

Cambios de comportamiento; mejor automantenimiento betes mellitus tipo II Síntomas depresivos

$$
\mathrm{N}=1
$$
del régimen diabético; resolución de síntomas depresivos; percepción de autoeficacia; realización de ejercicio físico
Nivel 3 de evidencia científica

$$
N=1
$$




\begin{tabular}{cc}
\hline $\begin{array}{c}\text { AUTOR / } \\
\text { LOCALIZACIÓN }\end{array}$ & OBJETIVO \\
\hline $\begin{array}{c}\text { Horowitz \& } \\
\text { Chang, 2003 }\end{array}$ & $\begin{array}{c}\text { Comprobar los efec- } \\
\text { tos de un programa } \\
\text { de redefinición de } \\
\text { estilos de vida en } \\
\text { Nueva York }\end{array}$ \\
& $\begin{array}{c}\text { lalud y el bienes- } \\
\text { intervención frente } \\
\text { al grupo de control } \\
\text { que recibe una pro- } \\
\text { grama usual }\end{array}$ \\
\hline
\end{tabular}

\section{Mountain et al., 2008}

Reino Unido
Comprobar la eficacia de una intervención de promoción de la salud basada en la ocupación en personas mayores

Examinar la efectividad de una intervención de revisión de vida mediante la escritura en persoNueva York nas mayores deprimidas en residencias

\section{DISEÑO Y NIVEL DE EVIDENCIA CIENTÍFICA}

\section{Cuantitativo \\ Ensayo aleatorizado}

Nivel 1 de evidencia científica

Cuantitativo y cualitativo

Ensayo pretest-posttest

Nivel 3 de evidencia científica

$$
\begin{gathered}
\text { Cuantitativo y } \\
\text { cualitativo } \\
\text { Ensayo } \\
\text { aleatorizado } \\
\text { Nivel } 1 \text { de evi- } \\
\text { dencia científica }
\end{gathered}
$$

\section{PRINCIPALES CONCLUSIONES} MUESTRA

\section{Edad $\geq 60$ años \\ MMSE $=24$ \\ Idioma inglés \\ Enfermedad crónica \\ Situación estable

$$
\mathrm{N}=28
$$

Grupo de control
$\mathrm{n}=16$

$$
\mathrm{n}=16
$$

Grupo de intervención

$$
\mathrm{n}=12
$$

Edad $\geq 60$ años Cúspide de bienestar Cúspide de fragilidad

No disfunción cognitiva

$$
\mathrm{N}=26
$$

$$
\begin{gathered}
\text { Edad } \geq 65 \text { años } \\
\text { Habla y escritura } \\
\text { inglesa } \\
\text { No demencia } \\
\mathrm{N}=45
\end{gathered}
$$

Grupo de control

$$
\mathrm{n}=22
$$

Grupo de intervención

\section{No diferencias estadís- ticamente significativas post intervención entre grupos en todas las variables analizadas; diferencias intragrupo en el posttest en el grupo de intervención en cuanto a una mejora de los síntomas depre- sivos y a una mejor puntuación en SF-36 (roles y dolor corporal) y en FSQ (dominios de salud)}

Beneficios individualizados; aumento de autoeficacia; aumento de la calidad de vida; no resultados estadísticamente significativos en depresión, cognición y dependencia

Diferencias estadísticamente significativas en síntomas depresivos; buena intervención para la práctica de TO 


\begin{tabular}{cc}
\hline $\begin{array}{c}\text { AUTOR / } \\
\text { LOCALIZACIÓN }\end{array}$ & OBJETIVO \\
\hline Clark et al., 2012 & $\begin{array}{c}\text { Determinar los efec- } \\
\text { tos que tiene una } \\
\text { intervención de TO } \\
\text { basada en estilos de } \\
\text { vida preventivos en } \\
\text { (California) } \\
\text { el bienestar físico y } \\
\text { mental y el nivel de } \\
\text { coste-efectividad }\end{array}$
\end{tabular}

\section{DISEÑO Y NIVEL CRITERIOS DE DE EVIDENCIA CIENTÍFICA}

\author{
Cuantitativo \\ Ensayo
} aleatorizado

Nivel 1 de evidencia científica coste-efectividad

\section{Cho et al., 2015 Ver los efectos que tiene un programa de entrenamiento grupal de estimu- lación cognitiva en la cognición, en la depresión y en la demencia}

Ensayo con grupo de control, no aleatorizado

Nivel 2 de evidencia científica

\section{Cuantitativo}

$$
\begin{gathered}
\text { Grupo de control } \\
\mathrm{n}=228
\end{gathered}
$$

Grupo de intervención $\mathrm{n}=232$

\section{Edad $>65$ años \\ Demencia}

Asistencia a centro de cuidados diarios

$$
\mathrm{N}=22
$$

Grupo de control

$$
\mathrm{n}=11
$$

Grupo de intervención

$$
\mathrm{n}=11
$$

Tanaka et al., 2015

Japón
Examinar el efecto de un programa grupal de psicoeducación en pacientes intra-hospitalarios con depresión

\section{Cuantitativo \\ Ensayo con gru- po de control, no aleatorizado \\ Nivel 2 de evi- dencia científica}

\section{PRINCIPALES CONCLUSIONES}

Diferencias estadísticamente significativas en dolor corporal, vitalidad, funcionamiento social, salud y funcionamiento mental, satisfacción con la vida y síntomas depresivos; no efecto en la función cognitiva; intervención rentable

No diferencias estadísticamente significativas entre grupos; mejoras en AVD, cognición y depresión en el posttest en el grupo de intervención
Edad media 63 años Episodio depresivo Sin trastorno de personalidad, dependencia alcohólica o discapacidad intelectual

$$
\mathrm{N}=82
$$

Grupo de control

$$
\mathrm{n}=37
$$

Grupo de intervención

$$
\mathrm{n}=45
$$

Diferencias no estadísticamente significativas en readmisión; mejor puntuación en SMSF, mejora de síntomas depresivos, recuperación y adherencia en grupo de intervención; eficacia en prevención de depresión por demostrar 


\section{DISEÑO Y NIVEL CRITERIOS DE DE EVIDENCIA INCLUSIÓN DE LA CIENTÍFICA MUESTRA \\ PRINCIPALES CONCLUSIONES}

\begin{tabular}{cc}
\hline $\begin{array}{c}\text { AUTOR / } \\
\text { LOCALIZACIÓN }\end{array}$ & OBJETIVO \\
\hline $\begin{array}{c}\text { Stevens-Rat- } \\
\text { chford, 1993 }\end{array}$ & $\begin{array}{c}\text { Evaluar la eficacia } \\
\text { de la reminiscencia } \\
\text { en la depresión y el } \\
\text { autoestima }\end{array}$ \\
& \\
McWha et al., & $\begin{array}{c} \\
\text { 2003 }\end{array}$ \\
& $\begin{array}{c}\text { Identificar los bene- } \\
\text { ficios de la actividad } \\
\text { terapéutica en grupo } \\
\text { en mujeres mayores } \\
\text { diagnosticadas de } \\
\text { depresión }\end{array}$ \\
&
\end{tabular}

Fine, 2001 Evaluar el efecto de las actividades de ocio en la depresión en los ancianos

\section{Cuantitativo \\ Ensayo aleatorizado}

Nivel 1 de evidencia científica

\section{Cuantitativo y cualitativo}

Ensayo con grupo de control, no aleatorizado

Nivel 2 de evidencia científica

\section{Grupo de intervención}

$$
\mathrm{n}=9
$$

\section{Criterios en común que tienen los artículos:
Edad > 65 años Depresión

$$
\mathrm{N}=8 \text { artículos }
$$

Nivel 1 de evidencia científica

\section{Gunnarsson et al., Evaluar la eficacia 2015 \\ Condados Suecos de The Tree Theme Method (interven- ción centrada en el cliente para desarro- Ilar estrategias que le ayuden en la vida diaria) en pacientes con depresión y ansiedad}

\section{Cuantitativo \\ Ensayo aletorizado \\ Nivel 1 de evi- dencia científica \\ Edad 18-65 años

$$
\begin{gathered}
\text { Depresión o } \\
\text { ansiedad }
\end{gathered}
$$ \\ Dificultad en AVD \\ No psicosis ni dete- rioro cognitivo

$$
\mathrm{N}=130
$$

Grupo de control

$$
\mathrm{n}=65
$$

Grupo de intervención

$$
\mathrm{n}=65
$$

Intervención beneficiosa para mujeres mayores deprimidas; expectativas entre grupos no estadísticamente significativas
Efecto beneficioso en la depresión
Intervención no estadísticamente significativa para depresión y autoestima 


\section{Discusión}

Aunque los síntomas depresivos son prevalentes en la población anciana y esta es cada vez más frecuente en la sociedad actual, y sabiendo que la TO es una disciplina con un campo de actuación amplio en esta población, el número de investigaciones y estudios realizados sobre este tema es muy escaso. A pesar de esta limitación, se han podido incluir en la revisión sistemática catorce artículos cuyo nivel de evidencia científica es moderado ya que se han incluido una revisión sistemática (nivel de evidencia 1), seis ensayos aleatorizados (nivel de evidencia 1), cuatro ensayos con grupo de control, no aleatorizados (nivel de evidencia 2) y dos ensayos pretest-posttest (nivel de evidencia 3), siendo el nivel 1 el mejor nivel de evidencia científica; aparte de un ensayo pretest-posttest con un enfoque cualitativo.

La revisión de historia de vida es una de las intervenciones que el terapeuta ocupacional desarrolla para tratar la depresión geriátrica. Así lo demuestran los estudios incluidos en esta revisión sistemática y planteados por Chippendale \& Bear-Lehman (2012) y Cooper (2013) que dejan ver como la revisión de historia de vida, haciendo uso de la escritura de forma estructurada, con una técnica específica de escritura y escribiendo sobre los ciclos vitales del usuario de forma cronológica, reduce la sintomatología depresiva que acontece en el mayor. Estudios anteriores corroboran también que estas técnicas son útiles para mejorar el estado de ánimo en comparación con otras formas de intervención (Mastel-Smith et al., 2006). Aunque sí es cierto que el estudio liderado por Cooper (2013) incluye una muestra demasiado estricta y no del todo alcanzable dentro de la población geriátrica, ya que entre los criterios de inclusión se encuentran que los participantes dispongan de habilidades como un razonamiento lógico, un amplio vocabulario y fluidez verbal. Así pues, en estudios posteriores, sería necesario poder incluir también a participantes con dificultades en estas habilidades. Del mismo modo, sería importante ampliar el número de participantes y dar al estudio un enfoque cuantitativo, y no solo cualitativo, para obtener mayor evidencia científica. Por contra, el estudio planteado por StevensRatchford (1993) encontró que su intervención basada en técnicas de reminiscencia no obtuvo efecto alguno sobre la sintomatología depresiva del mayor. Este hecho puede deberse a que al usar la técnica de reminiscencia la persona solo recuerda hechos relacionados con temas culturales de una época pasada (personajes, objetos, acontecimientos históricos, etc.), recuerdos que son menos significativos para la persona que los recuerdos autobiográficos. Además, este estudio debería incluir personas con mayores niveles de depresión, y no solo de o a 10 puntos en dicha escala. De la misma forma, se necesitan más estudios que evidencien los beneficios del método The Tree Theme Method (El Método del Árbol Temático) en la reducción de la sintomatología depresiva en personas mayores, ya que esta técnica solo está presentada como protocolo en el estudio de Gunnarsson et al. (2015) y aunque es cierto que hay estudios como el de Gunnarsson, Jansson, \& Eklund (2006) que demuestran que este método es útil para reducir la depresión, solo lo llevan a cabo con un solo caso y además en edad adulta y no mayor de 60 años.

Estudios incluidos en esta revisión sistemática como los de McWha et al. (2003) y Fine (2001) demuestran que las actividades de ocio y tiempo libre pueden ser utilizadas por los terapeutas ocupacionales para reducir los síntomas depresivos en personas mayores. Así lo evidencia un estudio que afirma que las actividades de ocio y tiempo libre repercuten en el bienestar psicológico de las personas mayores haciendo que se reduzcan sus síntomas depresivos, sus sentimientos de soledad, a la vez que aumente su nivel de satisfacción con la vida (García \& Gómez, 2003). No obstante, para mejorar este tipo de intervención el terapeuta ocupacional podría fijar su atención en las opiniones que tienen los usuarios sobre cuáles son las actividades de ocio y tiempo libre que pueden ayudar a su bienestar, cuáles son las más placenteras para ellos o conocer sus hobbies para ayudar a la selección de aquellas actividades que puedan mejorar su sintomatología depresiva. En definitiva, dar a la intervención un enfoque centrado en la persona. Ya estudios incluidos en esta revisión lo hacen, como el de Fine (2001) y el de Sood et al. (2003). Pero, este último estudio no obtiene diferencias estadísticamente significativas. Para conseguir diferencias estadísticamente significativas en sucesivos estudios se podría aumentar la muestra incentivando a los participantes a no abandonar durante el transcurso de la intervención. Para ello, sería beneficioso relacionar las actividades agradables de las que hace uso la intervención con la participación en roles ocupacionales significativos de la persona. De esta forma, el sentimiento de utilidad del que habla el Modelo de Ocupación Humana y por lo tanto, la responsabilidad y vinculación del usuario hacia la intervención se verían incrementados (Kielhofner, 2004). De igual forma, el estudio liderado por Mozley et al. (2007) 
no encuentra diferencias estadísticamente significativas haciendo uso de actividades creativas para disminuir la sintomatología depresiva en residencias. Aunque este estudio tenga un carácter principalmente exploratorio, en sucesivas investigaciones se debería aumentar el tamaño de la muestra y dar aleatorización a esta, hacer uso de instrumentos de medida más específicos y hacer que la intervención no esté restringida solo a un tipo de actividad (actividad creativa, en este caso), sino que, de nuevo, se adquiera un enfoque de intervención centrada en las preferencias de la persona.

En línea con el objetivo de este último artículo, en sucesivas investigaciones sería útil indagar en cómo la TO puede contribuir a reducir los síntomas depresivos que acontecen en personas mayores ante la institucionalización en residencias. Esta línea de investigación futura emerge debido a que el ámbito de trabajo más amplio de la TO en España es en residencias geriátricas y a que uno de los objetivos que el terapeuta ocupacional persigue en este ámbito es aumentar la calidad de vida y el bienestar de estos usuarios. Por lo que sabiendo este hecho, y conociendo que uno de los factores de riesgo para que las personas mayores padezcan sintomatología depresiva es el ingreso en residencias geriátricas, se debería investigar más en la labor que tiene el terapeuta ocupacional en el manejo de los sentimientos que produce la institucionalización geriátrica (Rodríguez \& Gómez, 2014).

Otro tipo de intervención de TO en el trabajo de la sintomatología depresiva en el anciano ha sido la participación en programas de promoción de estilos de vida más saludables que mejoren el bienestar entre los ancianos y prevengan enfermedades como la depresión. Mientras que los estudios de Clark et al. (2012) y Horowitz \& Chang (2003) encuentran efectos del Lifestyle Redesign Program en la reducción de sintomatología depresiva, el estudio llevado a cabo por Mountain et al (2008) no alcanza diferencias significativas en comparación con un grupo control. Este hecho puede deberse principalmente a que este estudio presenta una muestra de participantes inferior y no cuenta, por tanto, con suficiente potencia estadística. Es importante destacar que ambos estudios se realizaron en diferentes países, en Estados Unidos y en Inglaterra, con entornos culturales diferentes. Esta posible causa unida con la imposibilidad de modificar el Lifestyle Redesign Program para adaptarlo a la cultura donde se desarrolla podrían explicar las diferencias de los resultados.
La depresión en ancianos también puede ser consecuencia del establecimiento de un primer diagnóstico de enfermedad. Así ocurre en el estudio que presentan Haltiwanger \& Galindo (2013) donde la mujer que constituye la muestra del estudio está diagnosticada de diabetes y es a consecuencia de esto por lo que empieza a manifestar síntomas depresivos. La reducción de estos síntomas puede conseguirse con un mejor manejo de la enfermedad que da lugar a estos. Este estudio se centra en un caso único por lo que serían necesarios más estudios donde el terapeuta ocupacional trabaje con la práctica de habilidades de manejo de la enfermedad como medio para reducir los niveles de depresión y de esta forma poder generalizar los resultados. En este sentido, estudios con otro tipo de poblaciones también apuntan a la importancia del manejo de la enfermedad para mejorar la calidad de vida e incrementar el bienestar (Laliberte-Rudman, Yu, Scott, \& Pajouhandeh, 2000; Mancini, Hardiman, \& Lawson, 2005).

Por último, se han encontrado otras intervenciones usadas desde TO para trabajar los síntomas depresivos en el mayor. Estas han sido la Cognición y la Psicoeducación. Tanaka et al. (2015) haciendo uso de la Psicoeducación encuentran efectos en la reducción de los niveles de depresión. Sin embargo, hay matices que se podrían mejorar en sucesivas investigaciones, como el hacer uso de un test o escala específico de depresión. Por el contrario los autores Cho et al. (2015) no encuentran diferencias estadísticas entre grupos en los niveles de depresión haciendo uso del entrenamiento cognitivo como modo de intervención. Este hecho podría deberse principalmente al corto período de duración de la intervención y al reducido tamaño de la muestra.

Los posteriores estudios e investigaciones que aborden estas limitaciones, ayudarán a promulgar la labor de la TO en la sintomatología depresiva en la persona mayor. Sería importante que en estos estudios el terapeuta ocupacional incluya a participantes con diferentes niveles de sintomatología depresiva, desde leves a moderados y graves, con el objetivo de conocer con profundidad la efectividad de nuestra intervención en diferentes perfiles de personas. También, el terapeuta ocupacional podría hacer uso de estas intervenciones no solo para tratar los síntomas depresivos sino también para prevenirlos. 


\section{ReferenCias BibliográficAS}

Acebo, M. E. C., Mero, S. B. C., \& Bravo, W. M. D. (2016). El terapeuta ocupacional y su rol con pacientes geriátricos. Dominio de las Ciencias, 2(4), 60-71.

Ayala, A. G. (2007). La depresión en el anciano. Offarm, 26(9), 80-94.

Bettany-Saltikov, J. (2012). How to do a systematic literature review in nursing: a step-by-step guide. McGraw-Hill Education (UK).

Cabello, J. B. (2015). Lectura crítica de la evidencia clínica. Barcelona: Elsevier.

Chippendale, T., \& Bear-Lehman, J. (2012). Effect of life review writing on depressive symptoms in older adults: A randomized controlled trial. American Journal of Occupational Therapy, 66(4), 438-446.

Cho, M., Kim, D., Chung, J., Park, J., You, H., \& Yang, Y. (2015). Effects of a cognitive-enhancement group training program on daily living activities, cognition, and depression in the demented elderly. Journal of Physical Therapy Science, 27(3), 681-684. https://doi. org/10.1589/jpts.27.681

Ciucurel, C., \& Iconaru, E. I. (2012). A Case Study of Occupational Therapy for Poststroke Depression in Elderly. Procedia-Social and Behavioral Sciences, 46, 3865-3869. https://doi.org/10.1016/j. sbspro.2012.06.162

Clark, F., Jackson, J., Carlson, M., Chou, C.-P., Cherry, B. J., Jordan-Marsh, M., ... Azen, S. P. (2012). Effectiveness of a lifestyle intervention in promoting the well-being of independently living older people: results of the Well Elderly 2 Randomised Controlled Trial. Journal of Epidemiology \& Community Health, 66(9), 782-790. https:// doi.org/10.1136/jech.2009.099754

Cooper, P. (2013). Writing for depression in health care. The British Journal of Occupational Therapy, 76(4), 186-193. https://doi.org /10.4276/030802213X13651610908452

Fine, J. (2001). The effect of leisure activity on depression in the elderly: Implications for the field of occupational therapy. Occupational Therapy in Health Care, 13(1), 45-59.

García, M. A., \& Gómez, L. (2003). Efectos de los talleres de ocio sobre el bienestar subjetivo y la soledad en las personas mayores. Revista de Psicología Social, 18(1), 35-47.

Gunnarsson, A. B., Jansson, J.-Å., \& Eklund, M. (2006). The Tree Theme Method in Psychosocial Occupational Therapy: A Case Study. Scandinavian Journal of Occupational Therapy, 13(4), 229-240. https://doi.org/10.1080/11038120600772908

Gunnarsson, A. B., Wagman, P., Håkansson, C., \& Hedin, K. (2015). The Tree Theme Method ${ }^{\circledast}$ (TTM), an occupational therapy intervention for treating depression and anxiety: study protocol of a randomized controlled trial. BMC Psychology, 3(1). https://doi. org/10.1186/s40359-015-0097-9

Haltiwanger, E. P., \& Galindo, D. (2013). Reduction of Depressive Symptoms in an Elderly Mexican-American Female with Type 2 Diabetes Mellitus: A Single-Subject Study: Depression and Dia- betes. Occupational Therapy International, 20(1), 35-44. https:// doi.org/10.1002/oti.1338

Horowitz, B. P., \& Chang, P.-F.J. (2003). Promoting Well-Being and Engagement in Life Through Occupational Therapy Lifestyle Redesign: A Pilot Study Within Adult Day Programs. Topics in Geriatric Rehabilitation, 20(1), 46-58.

Kielhofner, G. (2004). Terapia ocupacional: modelo de ocupación humana: teoría y aplicación. Ed. Médica Panamericana.

Laliberte-Rudman, D., Yu, B., Scott, E., \& Pajouhandeh, P. (2000). Exploration of the perspectives of persons with schizophrenia regarding quality of life. The American Journal of Occupational Therapy: Official Publication of the American Occupational Therapy Association, 54(2), 137-147.

Leibold, M. L., Holm, M. B., Raina, K. D., Reynolds, C. F., \& Rogers, J. C. (2014). Activities and Adaptation in Late-Life Depression: A Qualitative Study. American Journal of Occupational Therapy, 68(5), 570. https://doi.org/10.5014/ajot.2014.011130

Mancini, M. A., Hardiman, E. R., \& Lawson, H. A. (2005). Making sense of it all: consumer providers' theories about factors facilitating and impeding recovery from psychiatric disabilities. Psychiatric Rehabilitation Journal, 29(1), 48-55.

Mastel-Smith, B., Binder, B., Malecha, A., Hersch, G., Symes, L., \& McFarlane, J. (2006). Testing therapeutic life review offered by home care workers to decrease depression among home-dwelling older women. Issues in Mental Health Nursing, 27(10), 1037-1049. https://doi.org/10.1080/01612840600943689

McWha, J. L., Pachana, N. A., \& Alpass, F. (2003). Exploring the therapeutic environment for older women with late-life depression: An examination of the benefits of an activity group for older people suffering from depression. Australian Occupational Therapy Journal, 50(3), 158-169.

Miralles, P. (2003, Febrero). La ocupación como método de tratamiento en salud mental. Comunicación presentada en el $4^{\circ}$ Congreso Virtual de Psiquiatría, Interpsiquis, Psiquiatria.com

Moher, D., Liberati, A., Tetzlaff, J., Altman, D. G., \& PRISMA Group. (2009). Preferred reporting items for systematic reviews and metaanalyses: the PRISMA statement. PLoS Medicine, 6(7), e1000097. https://doi.org/10.1371/journal.pmed.1000097

Mora, R. M. (2001). La depresión en el anciano. Terapia Ocupacional: Revista Informativa de La Asociación Profesional Española de Terapeutas Ocupacionales, (27), 87-96.

Mountain, G., Mozley, C., Craig, C., \& Ball, L. (2008). Occupational therapy led health promotion for older people: Feasibility of the Lifestyle Matters programme. The British Journal of Occupational Therapy, 71(10), 406-413.

Mozley, C. G., Schneider, J., Cordingley, L., Molineux, M., Duggan, S., Hart, C., ... Cruickshank, A. (2007). The Care Home Activity Project: Does introducing an occupational therapy programme reduce depression in care homes? Aging \& Mental Health, 17(1), 99-107. https://doi.org/10.1080/13607860600637810 
Rodríguez, E.J.F., \& Gómez, C. S. (2014). Estudio de la capacidad funcional y la calidad de vida relacionada con la salud en el adulto mayor institucionalizado. Revista electrónica de terapia ocupacional Galicia, TOG, (20 (Noviembre), 2.

Sevilla, J. G., \& Dallasta, R. M. (2006). Patologías psiquiátricas en el anciano: Detección y manejo por profesionales sanitarios y cuidadores. Revista Asturiana de Terapia Ocupacional, (3), 11-22.

Sood, J. R., Cisek, E., Zimmerman, J., Zaleski, E. H., \& Fillmore, H. H. (2003). Treatment of depressive symptoms during short-term rehabilitation: An attempted replication of the DOUR project. Rehabilitation PSychology, 48(1), 44-49. https://doi.org/10.1037/0090-5550.48.1.44

Stevens-Ratchford, R. G. (1993). The effect of life review reminiscence activities on depression and self-esteem in older adults. American Journal of Occupational Therapy, 47(5), 413-420.

Tanaka, S., Ishikawa, E., Mochida, A., Kawano, K., \& Kobayashi, M. (2015). Effects of Early-Stage Group Psychoeducation Programme for Patients with Depression: Group Psychoeducation Programme for Depression. Occupational Therapy International, 22(4), 195-205. https://doi.org/10.1002/oti.1397

Walsh, D., \& Downe, S. (2006). Appraising the quality of qualitative research. Midwifery, 22(2), 108-119. https://doi.org/10.1016/j. midw.2005.05.00 